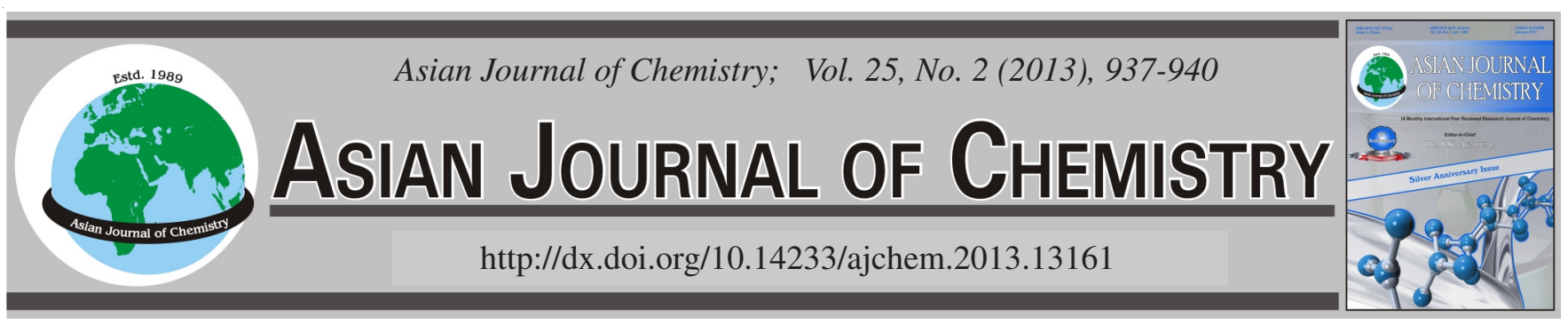

\title{
Characterization of Dominant Hydrogen Bonded Complex Structures
}

\author{
Aruna P. Maharolkar ${ }^{1, *}$, A.G. MurugkaR ${ }^{2}$, S.S. Patil ${ }^{2}$ and P.W. Khirade ${ }^{2}$
}

\begin{abstract}
${ }^{1}$ Microwave Research Lab, Department of Physics, Dr. Babasaheb Ambedkar Marathwada University, Aurangabad-431 004, India ${ }^{2}$ Department of Physics, Dr. Babasaheb Ambedkar Marathwada University, Aurangabad-431 004, India

*Corresponding author: E-mail: aruna_bamu@ @ediffmail.com
\end{abstract}

(Received: 2 December 2011;

Accepted: 27 August 2012)

AJC-12014

The density $(\rho)$, refractive index $\left(\mathrm{n}_{\mathrm{D}}\right)$, dielectric constant $\left(\varepsilon_{\mathrm{s}}\right)$ and relaxation time $(\tau)$ of binary mixture of allyl bromide $(\mathrm{AB})$ with $n$-butanol
including those of the pure liquids, were measured over the complete composition range at $283.15 \mathrm{~K}$. The experimental data is used to
calculate excess density $(\rho)^{\mathrm{E}}$, excess molar volumes $\left(\mathrm{v}_{\mathrm{m}}^{\mathrm{E}}\right)$, excess permittivity $\left(\varepsilon_{\mathrm{s}}^{\mathrm{E}}\right)$, excess inverse relaxation time $(1 / \tau)^{\mathrm{E}}$ and Bruggeman
factor. The excess parameters were fitted to Redlich-Kister equation. The variation of these parameters with composition and the effect of
bonding in new chemical species were discussed from the point of view of intermolecular interactions in these mixtures. The decrease in
excess molar volume $\left(\mathrm{v}_{\mathrm{m}}^{\mathrm{E}}\right)$ is attributed to the formation of hydrogen bonded associated species formed between unlike molecule.
Key Words: Excess molar refraction, Intermolecular interactions.

\section{INTRODUCTION}

A systematic investigation of volumetric and dielectric properties of mixed solvent systems and their dependence on composition do play a predictive role in a large spectrum of chemical, industrial and biological process. The importance of measuring the dielectric properties and volumetric properties of liquid mixtures lies in the fact that it provides valuable information about intermolecular interactions and dynamics of molecules at molecular levels ${ }^{1}$. Mixed solvents rather than single pure liquids are of utmost practical importance in most chemical and industrial processes as they provide a wide range of mixtures of two or more components in varying proportions. Now a day, polar class of liquids is attracting high interest because of strong intermolecular interaction amongst themselves and due to great solubility. The volumetric and dielectric properties of binary liquid mixtures are an invaluable type of data for the chemical engineer in the design and optimization of industrial processes. Furthermore, the simultaneous investigation and volume effects on mixing can be used as a powerful tool for the characterization of the intermolecular interactions present in the mixtures. Formerly we expressed the intermolecular associations among the components of alcohols with some other groups of liquids on the basis of dielectric studies only ${ }^{2-9}$. In this work we broaden the study by exploring properties like density and refractive index. Usual unassociated liquids are made up of large molecules bound by weakly directional van der Waals forces. The liquid selected for present study i.e. $n$-butanol on the contrary, is made up of small molecules powerfully bound by hydrogen bonds, in order of magnitude greater than van der Waals forces. Hydrogen bonding in butanol is supposed to be highly cooperative, i.e., interaction of butanol molecule with a group of hydrogen bonded molecules is more likely than interaction with a single molecule to give a dimer. Butanol has high solubility as well as polarity so it is used as a solvent in chemical industry.

\section{EXPERIMENTAL}

The chemicals used in the present paper ( $n$-butanol and allyl bromide) are of spectroscopic grade. The solutions were prepared at 11 different volume percentage of allyl bromide 0 to $100 \%$ in steps of $10 \%$ within $0.02 \%$ error limit.

Measurements: The basic TDR system consists of fast rising pulse generator, sampling unit and a wide band digitizing oscilloscope. A step voltage pulse produced by tunnel diode is detected at the sampler and displayed on the oscilloscope. This voltage pulse is propagated through a coaxial line. The cell which holds the sample is placed at the end of coaxial line. When the voltage pulse reaches the air-dielectric interface, a part of pulse is reflected and the rest is transmitted through the dielectric sample. The reflected part is recorded and reflection coefficient is determined. This reflection coefficient, after analysis provides the dielectric parameters. The setup used in this work is the HP54750A digitizing sampling oscilloscope and differential TDR module HP54754A with step generator (tunnel diode and built in circuitry $)^{10}$. A $200 \mathrm{mV}$ step pulse 
with $40 \mathrm{ps}$ rise time and at $250 \mathrm{KHz}$ pulse repetition rate passes through precision flexible coaxial $50 \mathrm{~W}$ transmission line. The waveform displayed on oscilloscope can be stored on 1.44 MB floppy disc drive available with the instrument.

The sample cell is a standard SMA connector with matched impedance with $3.5 \mathrm{~mm}$ outer diameter. The response of reflected pulses is observed in a time window of $5 \mathrm{~ns}$, which finds the most suitable time window for the liquids under study. The reflected pulses without sample $\left[\mathrm{R}_{1}(\mathrm{t})\right]$ and with sample $\left[\mathrm{R}_{\mathrm{x}}(\mathrm{t})\right]$ are digitized with 1024 points per waveform and stored in oscilloscope memory after averaging over 64 times. These reflected pulses are transferred to PC for further analysis.

The time dependent data were processed to obtain complex reflection coefficient spectra $\rho^{*}(\mathrm{w})$ over a frequency range $10 \mathrm{MHz}$ to $20 \mathrm{GHz}$ using Fourier transformation ${ }^{11,12}$ as:

$$
\rho^{*}(\omega)=\left(\frac{c}{j \omega d}\right)\left(\frac{p(\omega)}{q(\omega)}\right)
$$

where $\mathrm{p}(\omega)$ and $\mathrm{q}(\omega)$ are Fourier transforms of $\left[\mathrm{R}_{1}(\mathrm{t})-\mathrm{R}_{\mathrm{x}}(\mathrm{t})\right]$ and $\left[R_{1}(t)+R_{x}(t)\right]$ respectively, $c$ is the velocity of light, $\omega$ is the angular frequency, $\mathrm{d}$ is the effective pin length and $\mathrm{j}=\sqrt{-1}$.

The complex permittivity spectra were obtained from reflection coefficient spectra $\rho^{*}(\omega)$ by using bilinear calibration method ${ }^{13}$. The experimental values of $\varepsilon^{*}(\mathrm{w})$ are fitted with the Debye equation ${ }^{14}$.

$$
\varepsilon^{*}(\omega)=\varepsilon_{\infty}+\frac{\varepsilon_{\mathrm{s}}-\varepsilon_{\infty}}{1+j \omega \tau}
$$

where $\left(\varepsilon_{\mathrm{s}}\right)$ static dielectric constant, $\left(\varepsilon_{\infty}\right)$ high frequency dielectric constant and $(\tau)$ relaxation time are fitting parameters. A non-linear least square fit method ${ }^{15}$ was used to determine the values of dielectric parameters.

Refractive indices (at sodium D line) were measured by using Abbe Refractometer thermostated with constant water bath. Density is measured using Anton Paar Densitometer DMA 5000

\section{RESULTS AND DISCUSSION}

The density of the binary mixture of allyl bromide and $n$-butanol is decreasing as the volume fraction of $n$-butanol in the mixture is increasing. This is expected because density of pure allyl bromide is more than that of pure $n$-butanol. The variation of density as a function of volume fraction of $n$ butanol in the binary mixture indicates the existence of intermolecular interaction between the constituents of the mixture.

The refractive index $\left(n_{D}\right)$ is decreasing with increase in volume fraction of $n$-butanol in the mixture. This decrease in $\mathrm{R} \mathrm{I}\left(\mathrm{n}_{\mathrm{D}}\right)$ is further supported by the decrease in density of the mixture with increase in volume fraction of $n$-butanol. The value of dielectric constant increases with increase in volume fraction of $n$-butanol in the mixture (Table-1). This can be attributed to the increase in hydrogen bonding in the mixture. This is because there is dipole-dipole interaction in pure allyl bromide and strong hydrogen bonding as in pure $n$-butanol. As the percentage of $n$-butanol increases allyl bromide cooperates with $n$-butanol this cooperation leads to the formation of hydrogen bonding in the system. Relaxation time increases as concentration of $n$-butanol increases. Table- 1 indicates relaxation time as a function of volume fraction of $n$-butanol.

TABLE-1

STATIC DIELECTRIC CONSTANT, RELAXATION

TIME, DENSITY AND REFRACTIVE INDEX OF $n$-BUTANOL + ALLYL BROMIDE SYSTEM AT $283.15 \mathrm{~K}$

\begin{tabular}{ccccc}
\hline $\begin{array}{c}\text { Volume fraction } \\
\text { of butanol }\end{array}$ & $\begin{array}{c}\text { Dielectric } \\
\text { constant }\end{array}$ & $\begin{array}{c}\text { Relaxation } \\
\text { time }\end{array}$ & Density & RI \\
\hline 0 & 7.67 & 16.63 & 1.43653 & 1.472 \\
0.1 & 7.68 & 23.71 & 1.42643 & 1.464 \\
0.2 & 8.42 & 49.18 & 1.39985 & 1.456 \\
0.3 & 9.14 & 89.16 & 1.37701 & 1.444 \\
0.4 & 10.27 & 162.69 & 1.36678 & 1.440 \\
0.5 & 11.43 & 239.80 & 1.34178 & 1.432 \\
0.6 & 12.87 & 286.21 & 1.29988 & 1.426 \\
0.7 & 14.06 & 417.00 & 1.24312 & 1.421 \\
0.8 & 16.00 & 433.11 & 1.13113 & 1.416 \\
0.9 & 17.00 & 479.80 & 0.97325 & 1.405 \\
1.0 & 19.17 & 500.00 & 0.92063 & 1.400 \\
\hline
\end{tabular}

Fig. 1 showed that the excess density is positive, indicating the existence of intermolecular interaction.

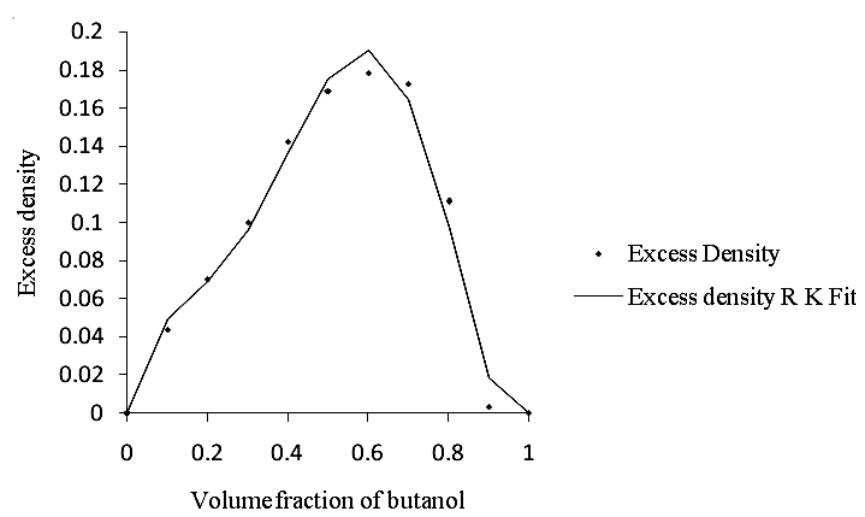

Fig. 1. Excess density of $n$-butanol with allyl bromide at $283.15 \mathrm{~K}$

The excess molar volumes $\mathrm{v}_{\mathrm{m}}{ }^{\mathrm{E}}$ were calculated by using the following relation ${ }^{16}$ :

$$
v_{m}^{\mathrm{E}}=x_{1} M_{1}\left(\frac{1}{\rho}-\frac{1}{\rho_{1}}\right)+x_{2} M_{2}\left(\frac{1}{\rho}-\frac{1}{\rho_{2}}\right)
$$

where $\mathrm{M}$ is the molecular mass; subscripts 1 and 2 stand for the pure components, allyl bromide and $n$-butanol respectively.

The variation of $\mathrm{v}_{\mathrm{m}}{ }^{\mathrm{E}}$ as volume fraction of allyl bromide is shown in Fig. 2. In pure $n$-butanol, molecules are hydrogen bonded. On addition allyl bromide, these molecules get in between the $n$-butanol molecules which further cooperates the hydrogen bonds of $n$-butanol molecules. This cooperation becomes the cause for decrease in molar volume of the mixture with increase in mole fraction of $n$-butanol in the mixture. Hence the excess molar volume is negative. Negative excess molar volume indicates hydrogen bonding in the system. This fact is further supported by the positive excess density, shown in Fig. 1.

Excess permittivity is given by:

$$
\varepsilon^{\mathrm{E}}=\varepsilon_{\mathrm{m}}-\left(\varepsilon_{\mathrm{A}} \mathrm{X}_{\mathrm{A}}+\varepsilon_{\mathrm{B}} \mathrm{X}_{\mathrm{B}}\right)
$$




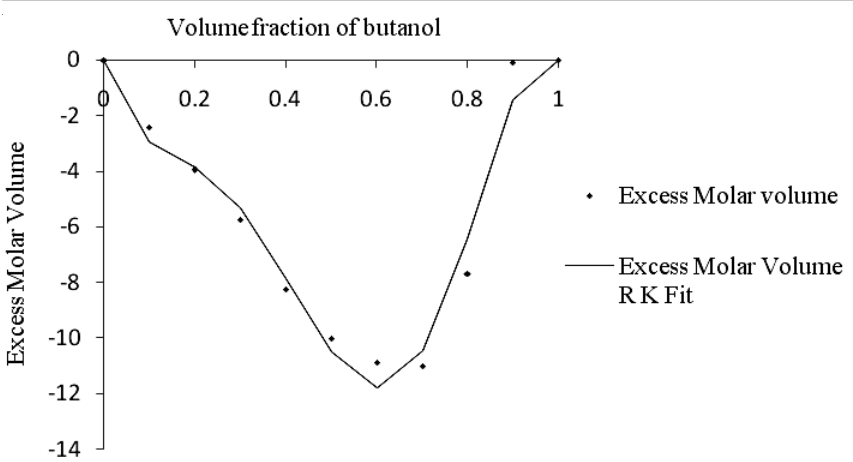

Fig. 2. Excess molar volume of allyl bromide $+n$-butanol

The variation of $\varepsilon_{\mathrm{s}}{ }^{\mathrm{E}}$ for increase in volume fraction of $n$-butanol in the binary mixture is shown in Fig. 3 which shows negative excess permittivity. The negative values of $\varepsilon_{\mathrm{s}}{ }^{\mathrm{E}}$ indicates that the solute and solvent interact in such a way that the effective dipole moment decreases. As concentration of $n$-butanol increases monomers and dimer combines to form polymers.

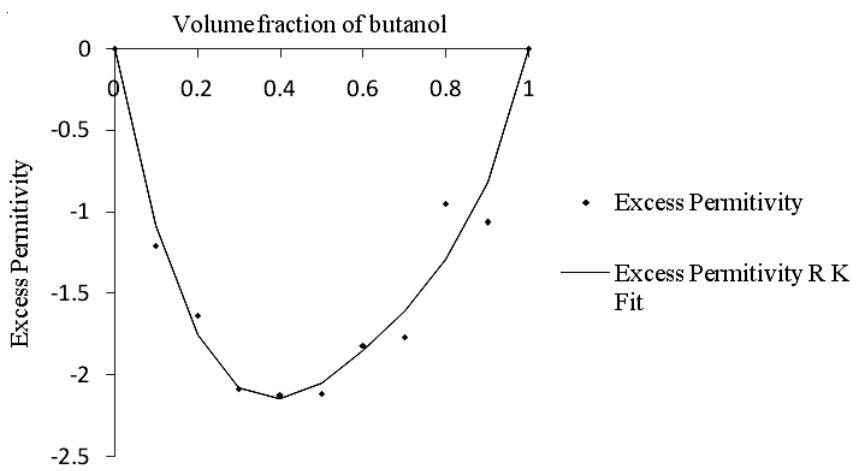

Fig. 3. Excess permittivity of allyl bromide $+n$-butanol

The excess inverse relaxation time is defined as:

$$
(1 / \tau)^{\mathrm{E}}=(1 / \tau)_{\mathrm{m}}-\left[(1 / \tau)_{\mathrm{A}} \mathrm{X}_{\mathrm{A}}+(1 / \tau)_{\mathrm{B}} \mathrm{X}_{\mathrm{B}}\right]
$$

where $(1 / \tau)^{\mathrm{E}}$ is excess inverse relaxation time. The information regarding the dynamics of solute-solvent interaction from this excess property can be predicted in the form of rotation of dipoles.

Fig. 4 describes negative excess inverse relaxation time.

$(1 / \tau)^{\mathrm{E}}<0$ indicates that the solute-solvent interaction produces a field such that the effective dipoles rotate slowly.

All excess parameters were fitted to the Redlich-Kister (RK) equation ${ }^{17}$. Table-2 shows $\mathrm{a}_{\mathrm{j}}$ coefficients and standard deviation $(\sigma)$ of excess parameters.

The modified Bruggeman factor is another parameter, which may be used as an indicator of inter-molecular interactions in liquid $A$ and $B$. The Bruggeman factor $f_{B}$ is given by

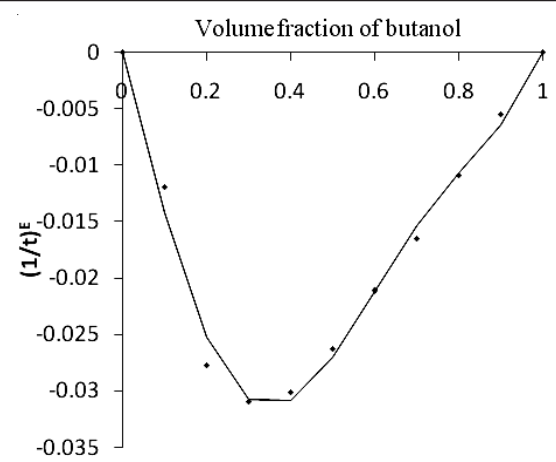

- Excess Inverse Relaxation time

Excess Inverse Relaxation time R K Fit

Fig. 4. Excess inverse relaxation time of allyl bromide $+n$-Butanol

$$
f_{B}=\left(\frac{\varepsilon_{s m}-\varepsilon_{s B}}{\varepsilon_{s A}-\varepsilon_{s B}}\right)\left(\frac{\varepsilon_{\mathrm{sA}}}{\varepsilon_{s m}}\right)^{\left(\frac{1}{3}\right)}=1-V
$$

Fig. 5 shows positive deviation from ideal line in Bruggeman plot indicates solute solvent interaction in the given system.

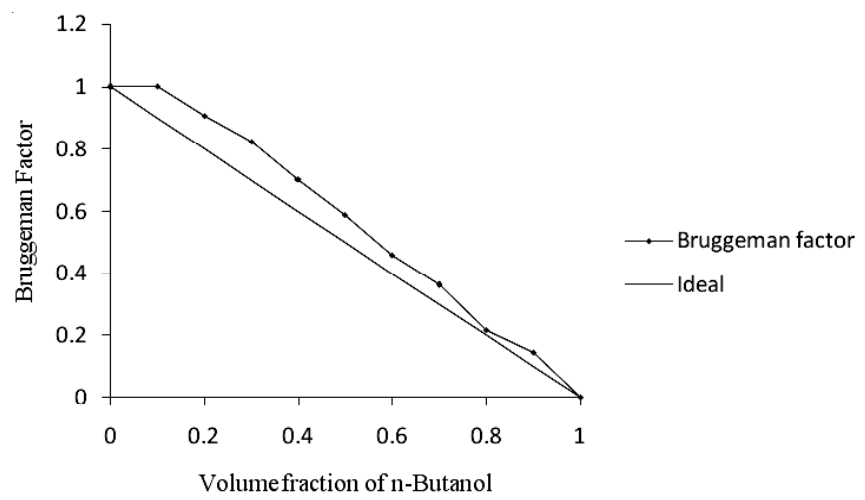

Fig. 5. Bruggeman factor of allyl bromide $+n$-butanol

\section{Conclusion}

The dielectric constant, relaxation time, density and refractive index of $n$-butanol + allyl bromide have been studied at $283.15 \mathrm{~K}$.

The values of refractive index and density are decreasing with increase in volume fraction of $n$-butanol. These two parameters are supporting each other. The value of $\varepsilon_{\mathrm{s}}$ increases as the concentration of $n$-butanol increases. This is due to increase in hydrogen bonding. The variations in refractive index, density and dielectric constant and relaxation time with volume fraction of $n$-butanol in the binary mixture of allyl bromide $+n$-butanol indicate the existence of intermolecular interaction. Negative excess molar volume i.e. contraction of volume indicates hydrogen bonding between solute and solvent. Non-linear deviation in Bruggeman plot also indicates solute solvent interaction in the system.

TABLE-2

$\mathrm{a}_{\mathrm{j}}$ COEFFICIENT (RK) AND STANDARD DEVIATION $(\sigma)$ OF EXCESS PARAMETERS

\begin{tabular}{lccccc}
\hline & $\mathrm{a}_{0}$ & $\mathrm{a}_{1}$ & $\mathrm{a}_{2}$ & $\mathrm{a}_{3}$ & $\sigma$ \\
\hline Excess density & 0.691236 & 0.656391 & -0.45354 & -1.30744 & $1.12 \times 10^{-2}$ \\
Excess molar volume & -41.373 & -46.4726 & 24.09655 & 85.40157 & 0.931366 \\
Excess permittivity & -8.26088 & 2.888767 & -3.38001 & -2.33047 & 0.184243 \\
Excess inverse relaxation time & -0.11015 & 0.100888 & $-3.68 \times 10^{-3}$ & $-8.29 \times 10^{-2}$ & $1.43 \times 10^{-3}$ \\
\hline
\end{tabular}




\section{REFERENCES}

1. P.B. Undre, S.N. Helambe, S.B. Jagdale, P.W. Khirade and S.C. Mehrotra, Pramana-J. Phys., 68, 851 (2007).

2. P.W. Khirade, A. Chaudhari, J.B. Shinde, S.N. Helambe and S.C. Mehrotra, J. Chem. Eng. Data, 44, 879 (1999).

3. B.G. Lone, P. Undre, S.S. Patil, P.W. Khirade and S.C. Mehrotra, J. Mol. Liq., 141, 47 (2008).

4. A. Chaudhari, P.W. Khirade, R. Singh, S.N. Helambe, N. Narain and S.C. Mehrotra, J. Mol. Liq., 82, 245 (1999).

5. A. Chaudhari, A. Das, G. Raju, H. Chaudhari, P.W. Khirade, N. Narain and S.C. Mehrotra, Proc. Natl. Sci. Counc. ROC(A), 25, 205 (2001).

6. K. Dharmalingam, K. Ramachandran, Sivagurunathan, B.P. Undre, P.W. Khirade and S.C. Mehrotra, Mol. Phys., 104, 2835 (2006).

7. P.W. Khirade, A. Chaudhary, J.B. Shinde, S.N. Helambe and S.C. Mehrotra, J. Solut. Chem., 28, 1031 (1999).

8. P. Sivagurunathan, K. Dharmalingam, K. Ramachandran, B.P. Undre, P.W. Khirade and S.C. Mehrotra, Phys. B, 387, 203 (2007).
9. P. Sivagurunathan, K. Dharmalingam, K. Ramachandran, B.P. Undre, P.W. Khirade and S.C. Mehrotra, Philos. Mag. Lett., 86, 291 (2006).

10. V.P. Pawar and S.C. Mehrotra, J. Mol. Liq., 95, 63 (2002).

11. C.E. Shannon, Proc IRE, 37, 10 (1949).

12. H.A. Samulan, Proc. IRE, 39, 175 (1951).

13. R.H. Cole, J.G. Berbarian, S. Mashimo, G. Chryssikos, A. Burns and E. Tombari, J Appl. Phys., 66,793 (1989).

14. P. Debye, Polar Molecules, Chemical Catalog, New York (1929); P.R. Bevington, Data Reduction and Error Analysis for the Physical Sciences, McGraw-Hill: New York (1969).

15. R. Baskaran and T.R. Kubendran, Chem. Thermodyn. Thermochem., 83, 350 (2009)

16. M. Lazarte, A.C. Gomez, Marigliano and H.N. Solimo, J. Solut. Chem., 33, 1549 (2004).

17. O. Redlich and A.T. Kister, Ind. Eng. Chem., 40, 345 (1948). 